CLINICAL STUDY

\title{
Increased preoperative glucose levels are associated with perioperative mortality in patients undergoing noncardiac, nonvascular surgery
}

\author{
Peter G Noordzij, Eric Boersma ${ }^{2}$, Frodo Schreiner, Miklos D Kertai ${ }^{1}$, Harm H H Feringa, Martin Dunkelgrun ${ }^{1}$, \\ Jeroen J Bax ${ }^{3}$, Jan Klein and Don Poldermans \\ Departments of Anesthesiology, ${ }^{1}$ Vascular Surgery, and ${ }^{2}$ Cardiology, Erasmus Medical Center, Dr Molewaterplein 40, 3015 GD, Rotterdam, \\ The Netherlands and ${ }^{3}$ Department of Cardiology, University Hospital Leiden, Leiden, The Netherlands
}

(Correspondence should be addressed to D Poldermans; Email: d.poldermans@erasmusmc.nl)

\begin{abstract}
Objective: To determine the relationship between preoperative glucose levels and perioperative mortality in noncardiac, nonvascular surgery.

Research design and methods: We performed a case-control study in a cohort of 108593 patients who underwent noncardiac surgery at the Erasmus MC during 1991-2001. Cases were 989 patients who underwent elective noncardiac, nonvascular surgery and died within 30 days during hospital stay. From the remaining patients, 1879 matched controls (age, sex, calendar year, and type of surgery) were selected. Information was obtained regarding the presence of cardiac risk factors, medication, and preoperative laboratory results. Preoperative random glucose levels $<5.6 \mathrm{mmol} / \mathrm{l}(110 \mathrm{mg} / \mathrm{dl})$ were normal. Impaired glucose levels in the range of $5.6-11.1 \mathrm{mmol} / \mathrm{l}$ were prediabetes. Glucose levels $\geq 11.1 \mathrm{mmol} / \mathrm{l}(200 \mathrm{mg} / \mathrm{dl})$ were diabetes.

Results: Preoperative glucose levels were available in 904 cases and 1247 controls. A cardiovascular complication was the primary cause of death in 207 (23\%) cases. Prediabetes glucose levels were associated with a 1.7-fold increased mortality risk compared with normoglycemic levels (adjusted odds ratio (OR) 1.7 and 95\% confidence interval (CI) 1.4-2.1; $P<0.001$ ). Diabetes glucose levels were associated with a 2.1-fold increased risk (adjusted OR 2.1 and 95\% CI 1.3-3.5; P<0.001). In cases with cardiovascular death, prediabetes glucose levels had a threefold increased cardiovascular mortality risk (adjusted OR 3.0 and 95\% CI 1.7-5.1) and diabetes glucose levels had a fourfold increased cardiovascular mortality risk (OR 4.0 and 95\% CI 1.3-12).

Conclusions: Preoperative hyperglycemia is associated with increased (cardiovascular) mortality in patients undergoing noncardiac, nonvascular surgery.
\end{abstract}

European Journal of Endocrinology 156 137-142

\section{Introduction}

Annually, approximately 26 million patients are scheduled for noncardiac surgery in the United States (1). Patients undergoing such surgery are at risk of perioperative cardiac events. In the study of Lee and colleagues, the incidence of major cardiac outcome in patients undergoing elective noncardiac surgery was $1.4 \%$, of which perioperative myocardial infarction (MI) was the most frequent contributor (2). The incidence of adverse cardiac events increases in patients at risk of cardiac disease prior to surgery (3). In this respect, diabetes mellitus has been associated with adverse cardiac outcome in noncardiac surgery $(2,4-6)$.

The prevalence of diabetes continues to increase worldwide, and is predicted to increase to 300 million by 2025 (7). Type 2 diabetes is the most prevalent form and accounts for approximately $90 \%$ of patients. Before the onset of type 2 diabetes, prediabetes patients exhibit a long asymptomatic period of increased glucose dysregulation (8). The relationship between diabetes and cardiovascular disease is thought to begin early in the progression from normal glucose tolerance to impaired glucose tolerance to diabetes $(9,10)$.

Impaired glucose metabolism is associated with adverse clinical outcome in the surgical and nonsurgical population (11-14). However, the relationship between preoperative glucose levels and perioperative mortality in noncardiac surgical patients has not adequately been defined. We hypothesized that preoperative hyperglycemia was associated with increased perioperative mortality in noncardiac, nonvascular surgical patients. 


\section{Methods}

\section{Study design}

We undertook a retrospective case-control study among 75581 patients above the age of 15 years who underwent 108593 noncardiac surgical procedures between January 1, 1991 and January 1, 2001 in the Erasmus MC, Rotterdam, The Netherlands. The computerized hospital information system was used to identify cases and controls. This system holds demographic and clinical data of all admitted patients, as well as information on the perioperative course.

\section{Selection of cases and controls}

The 2816 patients undergoing vascular surgery and the 129 patients with an American Society of Anesthesiologists (ASA) classification 5 (moribund, not expected to live $24 \mathrm{~h}$ irrespective of operation) were excluded. Candidate case subjects were the 1040 patients from the remaining population who died of any cause during surgery or during the hospital stay after surgery within 30 days.

We intended to select two controls for each case from the remaining patients. Cases and controls were matched according to the age (within an interval of \pm 5 years), gender, calendar year of surgery, and type of surgery. Surgical procedures were grouped according to a modified version of the American Heart Association/American College of Cardiology (AHA/ACC) classification (4). For 890 cases, two matching controls could be selected. For 99 other cases, only one matching control could be selected, whereas for 51 cases no matching controls could be selected. As a result, 989 cases were initially matched to 1879 controls. Our study population consisted of 904 cases and 1247 controls, in which data on preoperative plasma glucose levels were available.

\section{Data collection}

The computerized hospital database, patient medical records, nursing reports, surgical reports, anesthetic reports, and discharge letters were thoroughly analyzed by our investigators to obtain the following information on cases and controls; type of surgery, year of surgery, age, sex, diabetes, hypertension, family history of coronary artery disease (CAD), smoking, history of angina pectoris, MI, heart failure, coronary artery bypass grafting, percutaneous coronary intervention, cerebrovascular disease, chronic obstructive pulmonary disease, and renal insufficiency, as well as the ASA classification and the perioperative use of aspirin, oral anticoagulant therapy, $\beta$-blockers, nitrates, angiotensin-converting enzyme inhibitors, angiotensin 2 antagonists, statins, diuretics, prednisone, insulin, and oral antidiabetic medication.

\section{Preoperative glucose and diabetes status}

Patients were classified as having diabetes if their medical records showed documentation of a previous history of diabetes, or the use of oral antidiabetes medication or insulin at the time of hospital admission before the planned surgical procedure. Preoperative random glucose levels $<5.6 \mathrm{mmol} / \mathrm{l}(110 \mathrm{mg} / \mathrm{dl})$ were classified as normal. Impaired glucose levels in the range of 5.6-11.1 mmol/l were classified as prediabetes (15). Glucose levels $\geq 11.1 \mathrm{mmol} / \mathrm{l}(200 \mathrm{mg} / \mathrm{dl})$ were considered diabetes (16).

\section{Endpoint definition}

The hospital information system contains data regarding each patient's perioperative course. The vital status at hospital discharge was verified and documented for each patient. The occurrence of perioperative MI and clinically apparent strokes were reported. To obtain the cause of death, two investigators (MDK and DP) independently reviewed all available perioperative data, but were blind to preoperative characteristics. Cardiovascular death was defined as any death with a cardiovascular complication as the primary or secondary cause (according to the definitions of the World Health Organization), and included deaths following MI, cardiac arrhythmia, resuscitation, heart failure, or stroke. Noncardiovascular death was defined as any death with a principal noncardiovascular cause, including surgery-related bleeding complications, cancer, trauma, and infection. Sudden death in a previously stable patient was considered as cardiovascular. Events were counted until hospital discharge or 30 days after surgery, whichever day came first. We choose 30-day all-cause mortality and 30-day cardiovascular mortality as the endpoints of our study.

\section{Statistical analysis}

Continuous baseline data are described as median values and corresponding interquartile range (IQR), and dichotomous data are described as numbers and percentages. Differences between cases and controls were analyzed by Wilcoxon's tests for continuous variables, and $\chi^{2}$ tests for dichotomous variables.

We aimed to study to what extent preoperative glucose levels are associated with perioperative mortality. For this purpose, uni- and multivariable logistic regression analyses were applied, with preoperative glucose level as main exposure, and age, sex, type of surgery, a history of diabetes, hypertension, family history of CAD, smoking, angina pectoris, MI, heart failure, coronary artery bypass grafting, percutaneous coronary intervention, cerebrovascular disease, and renal insufficiency, ASA classification, and the perioperative use of aspirin, oral anticoagulant therapy, $\beta$-blockers, nitrates, angiotensin-converting enzyme 
inhibitors, angiotensin 2 antagonists, statins, diuretics, and corticosteroids as (potential) confounding factors. We report crude and adjusted odds ratios (ORs) and corresponding 95\% confidence intervals (CIs). All statistical tests were two-sided. $P<0.05$ was considered significant.

\section{Results}

\section{Preoperative glucose levels in relation to mortality}

Clinical baseline characteristics in cases and controls are presented in Table 1. Overall preoperative glucose levels were higher in cases than in controls (median $6.7 \mathrm{mmol} / \mathrm{l}$ and IQR $5.3-8.8 \mathrm{vs}$ median $5.7 \mathrm{mmol} / \mathrm{l}$ and IQR 4.8-7.2; $P<0.001$, Fig. 1). In cases, prediabetes and diabetes glucose levels were more frequently seen compared with controls (57 and $13 \%$ vs 50 and $5 \%$; $P<0.001$; Fig. 2, left panel).

Table 1 Baseline characteristics of cases and controls.

\begin{tabular}{|c|c|c|}
\hline & Cases & Controls \\
\hline No. of patients & 904 & 1247 \\
\hline \multicolumn{3}{|l|}{ Type of surgery } \\
\hline Low risk (\%) & 7 & 8 \\
\hline Low-intermediate risk (\%) & 24 & 21 \\
\hline Intermediate-high risk (\%) & 69 & 71 \\
\hline Age (years) & $64(48,74)$ & $65(51,75)$ \\
\hline Male gender (\%) & 61 & 63 \\
\hline \multicolumn{3}{|l|}{ Medical risk factors } \\
\hline Diabetes mellitus (\%) & 27 & $12^{\dagger}$ \\
\hline Hypertension (\%) & 20 & 17 \\
\hline Hypercholesterolemia (\%) & 17 & $7^{\dagger}$ \\
\hline $\begin{array}{l}\text { Family history of coronary } \\
\text { disease (\%) }\end{array}$ & 2 & 2 \\
\hline Current smoking (\%) & 14 & $9^{*}$ \\
\hline Angina pectoris (\%) & 14 & $9^{*}$ \\
\hline Myocardial infarction (\%) & 26 & $10^{\dagger}$ \\
\hline Heart failure (\%) & 11 & $3^{\dagger}$ \\
\hline $\begin{array}{l}\text { Percutaneous coronary } \\
\text { intervention (\%) }\end{array}$ & 2 & 2 \\
\hline $\begin{array}{l}\text { Coronary artery bypass } \\
\text { grafting }(\%)\end{array}$ & 3 & 4 \\
\hline Peripheral vessel disease & 11 & $6^{\dagger}$ \\
\hline Cerebrovascular disease & 7 & 6 \\
\hline $\begin{array}{l}\text { Chronic obstructive } \\
\text { pulmonary disease }\end{array}$ & 14 & $10^{\star}$ \\
\hline $\begin{array}{l}\text { Renal insufficiency } \\
\text { ASA classification }\end{array}$ & 35 & $16^{\dagger}$ \\
\hline I & 11 & 35 \\
\hline II & 18 & 35 \\
\hline III & 42 & 28 \\
\hline IV & 29 & 2 \\
\hline
\end{tabular}

${ }^{\star} P<0.01 ;{ }^{\dagger} P<0.001$.

${ }^{a}$ According to a modified version of the American Heart Association/American College of Cardiology classification: high risk, aortic; intermediate risk, abdominal, ear, nose, throat, neurologic, orthopedic, pulmonary, renal transplant, urologic, vascular, excluding aortic, and carotid; low risk, breast carotid, dental, endocrine, eye, gynecology, and reconstructive (4). Note that we excluded any vascular surgery, as a result we do not have patients in the high-risk category.

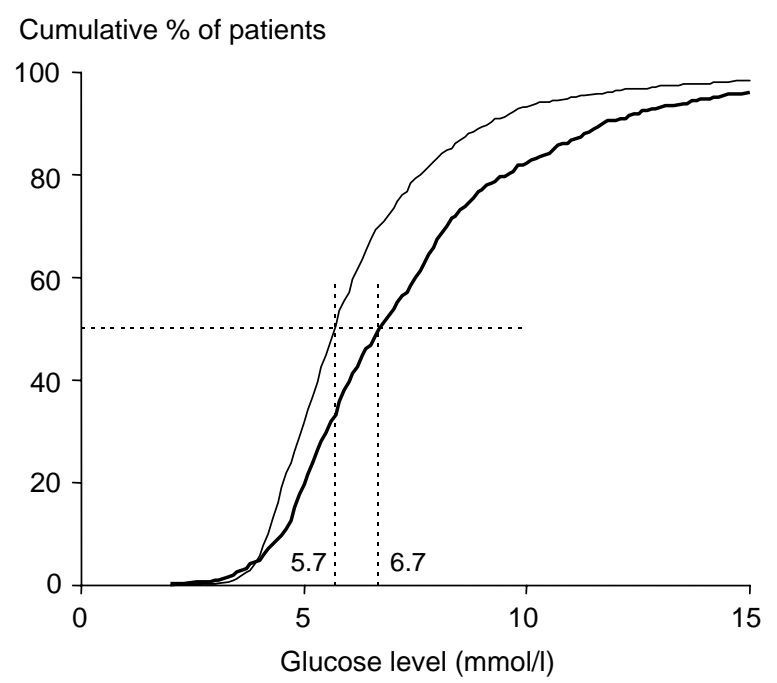

Figure 1 Cumulative distribution of glucose levels in cases (bold line) and controls.

After adjustment for a broad range of potential confounders, prediabetes glucose levels were associated with a 1.7 -fold increased mortality risk compared with normoglycemic levels (adjusted OR 1.7 and 95\% CI 1.42.1; $P<0.001$; Table 2). Diabetes glucose levels were associated with a 2.1-fold increased risk (adjusted OR 2.1 and $95 \%$ CI 1.3-3.5; $P<0.001)$.

In subjects with prediabetes glucose levels, a continuously increasing risk of perioperative mortality was observed along with increasing glucose concentration (Fig. 3). Each $1 \mathrm{mmol} / \mathrm{l}$ increase was associated with a $19 \%$ risk increase for mortality (adjusted OR 1.19 and 95\% CI 1.07-1.34; $P=0.002)$. A similar relationship was not observed
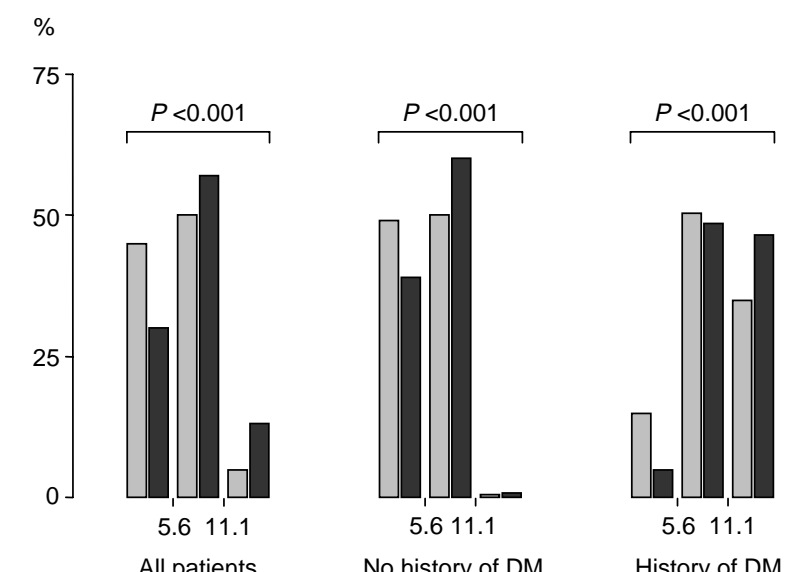

Figure 2 Incidence of normal, prediabetes, and diabetes preoperative glucose levels in cases (black) and controls (grey). Preoperative random glucose levels $<5.6 \mathrm{mmol} / \mathrm{l}(110 \mathrm{mg} / \mathrm{dl})$ were classified as normal. Impaired glucose levels in the range of 5.6-11.1 $\mathrm{mmol} / \mathrm{l}$ were classified as prediabetes. Glucose levels $\geq 11.1 \mathrm{mmol} / \mathrm{l}(200 \mathrm{mg} / \mathrm{dl})$ were considered diabetes. 
Table 2 Relation between perioperative mortality, preoperative glucose levels, and the use of antidiabetic medication.

\begin{tabular}{lccc}
\hline & Cases & Controls & $\begin{array}{c}\text { Adjusted odds ratio } \\
(95 \% \mathrm{Cl})^{\mathrm{a}}\end{array}$ \\
\hline $\begin{array}{l}\text { Glucose level } \\
\text { Normal }(<5.6 \mathrm{mmol} / \mathrm{l})\end{array}$ & $268(30 \%)$ & $561(50 \%)$ & 1 \\
$\quad$ Prediabetic $(5.6-11.1 \mathrm{mmol} / \mathrm{l})$ & $516(57 \%)$ & $626(50 \%)$ & $1.7(1.4-2.1)$ \\
$\quad$ Diabetic $(\geq 11.1 \mathrm{mmol} / \mathrm{l})$ & $120(13 \%)$ & $60(5 \%)$ & $2.1(1.3-3.5)$ \\
Medication & & $51(33 \%)$ & $0.27(0.14-0.53)$ \\
$\quad$ Insulin & $38(15 \%)$ & $42(27 \%)$ & $0.11(0.05-0.27)$ \\
$\quad$ Oral antidiabetic & $16(6 \%)$ & & \\
\hline
\end{tabular}

${ }^{a}$ Odds ratios were adjusted for a history of diabetes, type of surgery, age, sex, hypertension, family history of CAD, smoking, angina pectoris, myocardial infarction, heart failure, coronary artery bypass grafting, percutaneous coronary intervention, cerebrovascular disease, and renal insufficiency, as well as the ASA classification and the perioperative use of aspirin, oral anticoagulant therapy, $\beta$-blockers, nitrates, angiotensin-converting enzyme inhibitors, angiotensin 2 antagonists, statins, diuretics, and corticosteroids.

${ }^{\mathrm{b}}$ This analysis is based on 402 patients (247 cases and 155 controls) with a history of diabetes mellitus.

in subjects with diabetes glucose levels (adjusted OR 1.02 and $95 \%$ CI $0.91-1.15 ; P=0.71$ ).

\section{Preoperative glucose levels and cardiovascular mortality}

A cardiovascular complication was the primary cause of death in $207(23 \%)$ cases. The median glucose level of these cases was $7.1 \mathrm{mmol} / \mathrm{l}$ (IQR 5.3-9.0), significantly higher than the value in their matching controls $(N=$ 320; median $5.8 \mathrm{mmol} / \mathrm{l}$ and IQR 4.8-7.4; $P<0.001$ ). Prediabetes glucose levels were associated with a threefold increased cardiovascular mortality risk compared with normoglycemic levels (adjusted OR 3.0 and 95\% CI 1.7-5.1). Diabetes glucose levels were associated with a fourfold increased cardiovascular mortality risk (OR 4.0 and 95\% CI 1.3-12). Again, in subjects with prediabetes glucose levels, a continuously increasing risk of perioperative cardiovascular mortality was observed along with increasing glucose levels, however, this relationship did not reach statistical significance (adjusted OR 1.24 per $\mathrm{mmol} / \mathrm{l}$ and $95 \%$ CI $0.94-1.64 ; P=0.12$ ).

\section{History of diabetes mellitus and diabetes medication in cases and controls}

A history of diabetes mellitus was present in 402 patients (247 cases and 155 controls). Patients with a history of diabetes had significantly higher glucose levels than those without such history (median $5.7 \mathrm{mmol} / \mathrm{l}$ and IQR $4.8-7.0$ vs median $10.5 \mathrm{mmol} / \mathrm{l}$ and IQR $7.8-12.6 ; P<0.001)$. In patients without a history of diabetes, cases were more often classified with prediabetes glucose levels than controls (60 vs 50\%; $P<$ 0.001; Fig. 2, middle panel). In those with a history of diabetes, the percentage of cases and controls with prediabetes glucose levels was similar, but cases more often showed diabetes glucose levels compared with controls (47 vs $35 \% ; P<0.001$; Fig. 2, right panel).

In patients with a history of diabetes, $20 \%$ used insulin, $12 \%$ used oral antidiabetes medication, and $2 \%$ used both therapies. The use of any antidiabetes medication was associated with reduced glucose levels compared with patients without antidiabetes medication (median $9.3 \mathrm{mmol} / \mathrm{l}$ and IQR $6.3-12.7 \mathrm{vs}$ median $10.8 \mathrm{mmol} / \mathrm{l}$ and IQR 8.3-12.6; $P=0.003$ ). Insulin was used by $15 \%$ of cases vs $33 \%$ of controls $(P<0.001)$, oral antidiabetes medication was used by $6 \%$ of cases vs $27 \%$ of controls $(P<0.001)$. After adjustment for a broad range of potential confounders (Table 2), insulin and oral antidiabetes treatment were associated with reduced risk of perioperative mortality. When limited to the 207 cases with cardiovascular death and their matching controls, insulin therapy

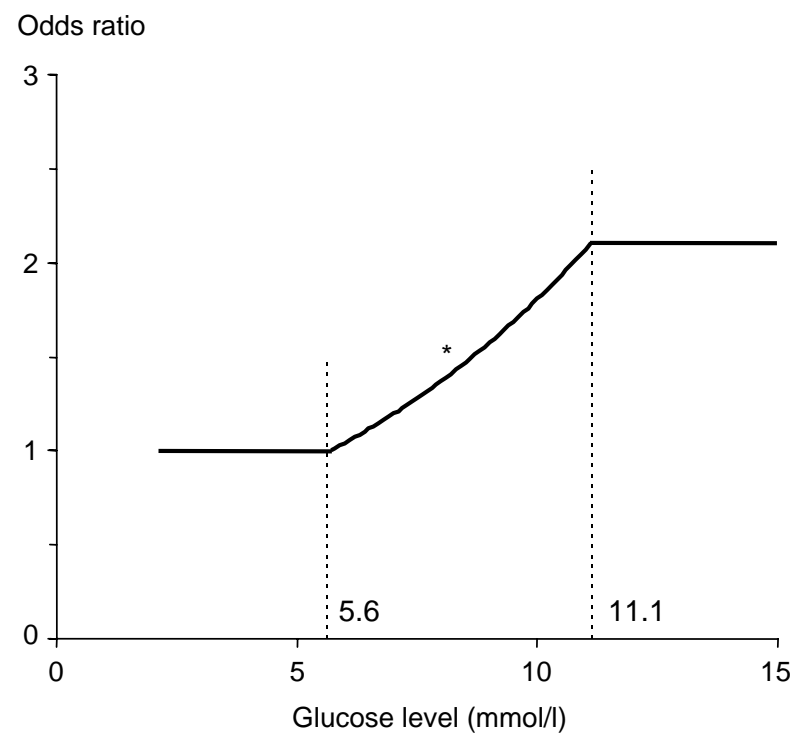

Figure 3 Relation between glucose level and risk (odds ratio) for perioperative mortality. Odds ratios are adjusted for history of diabetes, type of surgery, age, sex, hypertension, family history of $\mathrm{CAD}$, smoking, angina pectoris, myocardial infarction, heart failure, coronary artery bypass grafting, percutaneous coronary intervention, cerebrovascular disease, and renal insufficiency, as well as the ASA classification and the perioperative use of aspirin, oral anticoagulant therapy, $\beta$-blockers, nitrates, angiotensin-converting enzyme inhibitors, angiotensin 2 antagonists, statins, diuretics, and corticosteroids. ${ }^{*}$ Odds ratio for perioperative mortality is $1.19(95 \%$ Cl 1.1-1.3) per $\mathrm{mmol} / \mathrm{l}$ increase of glucose level. 
(adjusted OR 0.04 and 95\% CI 0.01-0.22) as well as oral antidiabetes medication (adjusted OR 0.04 and 95\% CI 0.01-0.26) remained associated with lower perioperative risk.

\section{Discussion}

In this case-control study of patients undergoing noncardiac, nonvascular surgery, elevated preoperative glucose levels were associated with increased perioperative mortality. Glucose levels in the diabetes range were associated with a more than twofold all-cause mortality increase, and a fourfold cardiovascular mortality increase, compared with normoglycemic levels. Importantly, glucose levels in the prediabetes range were also associated with increased risk for both endpoints. Antidiabetes medication was associated with a reduction in all-cause mortality as well as cardiovascular mortality.

Prediabetes glucose levels in patients without a history of diabetes were associated with increased perioperative (cardiovascular) death. This can be explained by a number of possible mechanisms. First, the relationship between diabetes and cardiovascular disease is thought to start early in the progression from normal glucose tolerance to impaired glucose metabolism, and from impaired glucose tolerance to diabetes $(9,10)$. As a result of reduced insulin tolerance, and subsequent hyperglycemia, prediabetes patients may be at risk for cardiovascular disease. Secondly, patients with impaired glucose regulation are more frequently diagnosed with hypertension, obesity, and dyslipidemia, compared with normoglycemic patients. This cluster of risk factors, also known as the metabolic syndrome, is associated with adverse cardiovascular outcome (17).

Perioperative MI is the most frequent fatal cardiovascular complication during noncardiac surgery $(2,3)$, however, the pathophysiology underlying perioperative MI is not completely clear. During surgery, the patient is exposed to a stress response, which includes a catecholamine surge with associated hemodynamic stress, vasospasm, reduced fibrinolytic activity, platelet activation, and consequent hypercoagulability (18). This stress state contributes to coronary plaque instability and rupture in patients with (asymptomatic) atherosclerotic coronary disease, which leads to thrombus formation and subsequent vessel occlusion (18). Impaired glucose metabolism seems to be associated with increased inflammation of preexistent atheromatous coronary plaques, resulting in intraplaque hemorrhage, and possible plaque rupture leading to MI $(19,20)$. In addition, in patients with fixed stenotic coronary lesions, increased heart rate, and contractility may induce an oxygen supply and demand mismatch leading to myocardial ischemia and eventually MI (18).

In patients with a known history of diabetes, preoperative hyperglycemia was associated with worse perioperative outcome. Previous studies in patients undergoing noncardiac surgery have identified a history of diabetes as a risk factor for perioperative cardiac events, without taking glucose levels into account (2, 4-6). Stratton and colleagues (21) showed that in patients with known diabetes, the risk of atherosclerotic cardiovascular disease increased with increasing plasma glucose concentration, each $1 \%$ increase in $\mathrm{HbA} 1 \mathrm{c}$ level was associated with a $14 \%$ increase in the incidence of fatal and nonfatal MI. In patients with diabetes undergoing coronary artery bypass graft surgery, perioperative control of glucose levels was associated with a reduction in episodes of recurrent ischemia and an improved survival in the first 2 years after surgery (23). Oral antidiabetes medication as well as insulin therapy resulted in a significant reduction of perioperative mortality. In previous studies, glycemic control by insulin therapy has been shown to result in marked improvement in cardiac survival in hyperglycemic nondiabetes patients and diabetes patients with acute coronary syndromes, MI, and those who have undergone recent cardiac surgery $(11,22,23)$. The switch from free fatty acid myocyte metabolism to glucose metabolism in ischemic myocardium may be an important therapeutic intervention during ischemia (24). However, the protective effect is not entirely clear; is benefit derived from glycemic control or infused insulin or both? In our study population, the effect of oral antidiabetes medication was similar to that of insulin treatment, and both therapies were associated with significantly lower preoperative glucose levels in controls compared with cases.

The results of our study suggest that screening for glucose dysregulation in surgical patients should be part of standard preoperative testing. The preoperative metabolic state, as measured by random blood glucose measurements, identifies patients at risk for perioperative cardiovascular events. Randomly determined glucose levels have been shown to be an efficient test to identify patients with prediabetes and undiagnosed diabetes (15). As it seems to become increasingly clear that impaired glucose metabolism and the prediabetes state are associated with adverse clinical outcomes, we suggest that patients with preoperative glucose levels above $5.6 \mathrm{mmol} / \mathrm{l}$ are further screened for associated cardiovascular risk factors. In this respect, the type of surgical procedure, patient clinical characteristics, further noninvasive test results, and additional medical therapy may improve perioperative patient management $(4,25,26)$.

Our study has several limitations that are common with any case-control study relying on retrospective data collection. First, information on patient characteristics might have been missed because of observer bias prejudice. Secondly, multivariable adjustment for potential confounders is obviously limited to the available data elements. Unknown, unmeasured confounders might still be present. Thirdly, the number of 
patients on oral antidiabetes medication and insulin therapy was limited and data on perioperative glucose levels were not available. Therefore, we would like to emphasize that this early evidence needs confirmation by adequately powered randomized clinical trials.

\section{Conclusion}

This case-control study provides evidence that preoperative hyperglycemia is associated with increased preoperative mortality.

\section{References}

1 DeFrances CJ \& Hall MJ. 2002 National Hospital Discharge Survey. Advance Data 2004342 1-29.

2 Lee TH, Marcantonio ER, Mangione CM, Thomas EJ, Polanczyk CA, Cook EF, Sugarbaker DJ, Donaldson MC, Poss R, Ho KK, Ludwig LE, Pedan A \& Goldman L. Derivation and prospective validation of a simple index for prediction of cardiac risk of major noncardiac surgery. Circulation 19991001043 1049.

3 Devereaux PJ, Goldman L, Cook DJ, Gilbert K, Leslie K \& Guyatt GH. Perioperative cardiac events in patients undergoing noncardiac surgery: a review of the magnitude of the problem, the pathophysiology of the events and methods to estimate and communicate risk. Canadian Medical Association Journal 2005173 $627-634$.

4 Boersma E, Kertai MD, Schouten O, Bax JJ, Noordzij P, Steyerberg EW, Schinkel AF, van Santen M, Simoons ML, Thomson IR, Klein J, van Urk H \& Poldermans D. Perioperative cardiovascular mortality in noncardiac surgery: validation of the Lee cardiac risk index. American Journal of Medicine 2005118 1134-1141.

5 Mangano DT, Browner WS, Hollenberg M, London MJ, Tubau JF \& Tateo IM. Association of perioperative myocardial ischemia with cardiac morbidity and mortality in men undergoing noncardiac surgery. The Study of Perioperative Ischemia Research Group. New England Journal of Medicine 1990323 1781-1788.

6 Eagle KA, Berger PB, Calkins H, Chaitman BR, Ewy GA, Fleischmann KE, Fleisher LA, Froehlich JB, Gusberg RJ, Leppo JA, Ryan T, Schlant RC, Winters WL Jr, Gibbons RJ, Antman EM, Alpert JS, Faxon DP, Fuster V, Gregoratos G, Jacobs AK, Hiratzka LF, Russell RO \& Smith SC Jr. ACC/AHA guideline update for perioperative cardiovascular evaluation for noncardiac surgery - executive summary a report of the American College of Cardiology/American Heart Association Task Force on Practice Guidelines (Committee to Update the 1996 Guidelines on Perioperative Cardiovascular Evaluation for Noncardiac Surgery). Circulation 2002105 1257-1267.

7 King H, Aubert RE \& Herman WH. Global burden of diabetes, 1995-2025. Prevalence, numerical estimates, and projections. Diabetes Care 199821 1414-1431.

8 Deedwania PC \& Fonseca VA. Diabetes, prediabetes, and cardiovascular risk: shifting the paradigm. American Journal of Medicine 2005118 939-947.

9 Is the current definition for diabetes relevant to mortality risk from all causes and cardiovascular and noncardiovascular diseases? Diabetes Care 200326 688-696.

10 Levitan EB, Song Y, Ford ES \& Liu S. Is nondiabetic hyperglycemia a risk factor for cardiovascular disease? A meta-analysis of prospective studies Archives of Internal Medicine $2004 \mathbf{1 6 4}$ 2147-2155.
11 Lazar HL, Chipkin SR, Fitzgerald CA, Bao Y, Cabral H \& Apstein CS. Tight glycemic control in diabetic coronary artery bypass graft patients improves perioperative outcomes and decreases recurrent ischemic events. Circulation 2004109 1497-1502.

12 van den Berghe G, Wouters P, Weekers F, Verwaest C, Bruyninckx F, Schetz M, Vlasselaers D, Ferdinande P, Lauwers P \& Bouillon R. Intensive insulin therapy in the critically ill patients. New England Journal of Medicine 2001 345 1359-1367.

13 Van den Berghe G, Wilmer A, Hermans G, Meersseman W, Wouters PJ, Milants I, Van Wijngaerden E, Bobbaers H \& Bouillon R. Intensive insulin therapy in the medical ICU. New England Journal of Medicine $2006 \mathbf{3 5 4} 449-461$.

14 Kosiborod M, Rathore SS, Inzucchi SE, Masoudi FA, Wang Y, Havranek EP \& Krumholz HM. Admission glucose and mortality in elderly patients hospitalized with acute myocardial infarction: implications for patients with and without recognized diabetes. Circulation $20051113078-3086$.

15 Zhang P, Engelgau MM, Valdez R, Cadwell B, Benjamin SM \& Narayan KM. Efficient cutoff points for three screening tests for detecting undiagnosed diabetes and pre-diabetes: an economic analysis. Diabetes Care 200528 1321-1325.

16 Report of the expert committee on the diagnosis and classification of diabetes mellitus. Diabetes Care 200326 S5-S20.

17 Nigam A, Bourassa MG, Fortier A, Guertin MC \& Tardif JC. The metabolic syndrome and its components and the long-term risk of death in patients with coronary heart disease. American Heart Journal 2006151 514-521.

18 Libby P, Ridker PM \& Maseri A. Inflammation and atherosclerosis. Circulation 2002105 1135-1143.

19 Bell DS. Inflammation, insulin resistance, infection, diabetes, and atherosclerosis. Endocrine Practice 20006 272-276.

20 Moreno PR \& Fuster V. New aspects in the pathogenesis of diabetic atherothrombosis. Journal of the American College of Cardiology 200444 2293-2300.

21 Stratton IM, Adler AI, Neil HA, Matthews DR, Manley SE, Cull CA, Hadden D, Turner RC \& Holman RR. Association of glycaemia with macrovascular and microvascular complications of type 2 diabetes (UKPDS 35): prospective observational study. British Medical Journal 2000321 405-412.

22 Capes SE, Hunt D, Malmberg K \& Gerstein HC. Stress hyperglycaemia and increased risk of death after myocardial infarction in patients with and without diabetes: a systematic overview. Lancet $2000355773-778$.

23 Gerstein HC, Pais P, Pogue J \& Yusuf S. Relationship of glucose and insulin levels to the risk of myocardial infarction: a case-control study. Journal of the American College of Cardiology 199933 612-619.

24 McNulty PH. Comparison of local and systemic effects of insulin on myocardial glucose extraction in ischemic heart disease. American Journal of Physiology. Heart and Circulatory Physiology 2000278 H741-H747.

25 Noordzij PG, Boersma E, Bax JJ, Feringa HH, Schreiner F, Schouten O, Kertai MD, Klein J, van Urk H, Elhendy A \& Poldermans D. Prognostic value of routine preoperative electrocardiography in patients undergoing noncardiac surgery. American Journal of Cardiology 200697 1103-1106.

26 Boersma E, Poldermans D, Bax JJ, Steyerberg EW, Thomson IR, Banga JD, van De Ven LL, van Urk H \& Roelandt JR. Predictors of cardiac events after major vascular surgery: role of clinical characteristics, dobutamine echocardiography, and beta-blocker therapy. Journal of the American Medical Association 2001285 1865-1873.

Received 6 June 2006

Accepted 31 October 2006 